\title{
Is Change a Fact of Life?
}

\author{
Melanie Oakley
}

Editor-in-Chief

C HANGE is what makes nursing exciting; as a profession, we have moved on from when I trained as a nurse in 1979 , which to some readers may seem a long time ago, but for me it seems like yesterday. I trained in a time when you were taught to accept the instructions given to you and not question the validity of them. However, as a practitioner I have watched and taken an active role in the evolution of nursing. Nurses now are taught to reflect upon practice and question the care given to patients. This is a good thing because it can only benefit the patient who must always be our first priority.

I work in a nurse-led Post Anaesthetic Care Unit, but what does this mean in real terms? We have a philosophy within the centre I work that if you come to a manager with a problem you must also come with a possible solution. This way all staff are encouraged to take ownership of issues and be involved in problem solving. Staff have said to me that working in the unit is different from where they have worked before. Their opinion is respected and they are able to think 'outside the box', something that is actively encouraged.

So what is a nurse-led unit? In the centre we do not have junior doctors, instead, we have nurses whose skills have been developed to a standard where they can physically assess patients, order and interpret X-rays, and make decisions on the treatment patients will receive. We have an intensivist on call 24 hours a day, who actively encourage nurses within the centre to work autonomously, and are on hand for consultation and educational support.

In a Post Anaesthetic Care Unit, working autonomously is not new; however, we have been able to take this one step further. The staff come from a combination of backgrounds, primarily recovery and ITU/HDU and the synergy between the two has been astounding. Reciprocal teaching has gone on and each group respects the skills of the other, and what could have been a difficult alliance of staff coming from two different paradigms has worked successfully to the benefit of the patient. An example of this is our fluid challenge policy. All patients are written for a fluid challenge if required, nurses make the decision as to whether to administer this and when. The nurses with HDU/TTU experience are used to making decisions like this whereas the recovery nurses have found this slightly harder, but there is a definite shift in confidence as the staff are supported in their decisions. A standard reply when staff query something is to say, 'tell me what you think you should do?'

The change process I have gone through in the last three months has been dramatic and part of my philosophy is never be too humble to say you do not know something. I would much rather someone some said to me they were unsure than went ahead and did something when they did not really know what they were doing. To me, that is safe practice. Many nurses I have met are resistant to change, but my message is if we do not embrace it, how is our practice going to change? 